\title{
ON CERTAIN GROUPS OF BIRATIONAL CONTACT TRANSFORMATIONS*
}

\author{
J. M. FELD
}

1. Introduction. Let $x_{i}$ and $u_{i},(i=1,2,3)$, be the projective coordinates in plane $x u$ of a point and line, respectively, and $y_{i}$ and $v_{i}$ the coordinates of a point and line in plane $y v$. The planes may be superimposed. Let $s$ be a transformation in mixed coordinates

$$
\rho y_{i}=\phi_{i}(x, u), \quad \sigma v_{i}=\psi_{i}(x, u), \quad i=1,2,3,
$$

where the $\phi_{i}$ and the $\psi_{i}$ are polynomials homogeneous in the $x_{i}$ of degrees $a$ and $c$, respectively, and are also polynomials in the $u_{i}$ of degrees $b$ and $d$, respectively. This transformation is said to be birational if from (1) it is possible to obtain the inverse $s^{-1}$ given by

$$
\rho^{\prime} x_{i}=\phi_{i}^{\prime}(y, v), \quad \sigma^{\prime} u_{i}=\psi_{i}^{\prime}(y, v), \quad i=1,2,3,
$$

where the $\phi_{i}^{\prime}$ and $\psi_{i}^{\prime}$ are polynomials homogeneous in the $y_{i}$ of degrees $a^{\prime}$ and $c^{\prime}$, and in the $v_{i}$ of degrees $b^{\prime}$ and $d^{\prime}$, respectively, and

$$
\rho^{\prime \prime} y_{i}=\phi_{i}\left(\phi^{\prime}, \psi^{\prime}\right), \quad \sigma^{\prime \prime} v_{i}=\psi_{i}\left(\phi^{\prime}, \psi^{\prime}\right) .
$$

This transformation is a line element transformation $\dagger$ if each of the equations

$$
\sum u_{i} x_{i}=0, \quad \sum v_{i} y_{i}=0
$$

implies the other. An element transformation is a birational contact transformation if, in addition to the above requirements, each of the two systems of equations

$$
\sum x_{i} d u_{i}=\sum u_{i} d x_{i}=0
$$

and

$$
\sum y_{i} d v_{i}=\sum v_{i} d y_{i}=0
$$

implies the other. $\ddagger$

Simple examples of birational contact transformations in the plane

* Presented to the Society, February 26, 1938.

$\dagger$ A line element transformation is not a contact transformation unless it preserves unions.

$\ddagger$ The representation of line elements by means of six coordinates $x_{i}, u_{i}$ is due to Clebsch who also gave the necessary and sufficient conditions that (1) be a contact transformation. Clebsch-Lindemann-Benoist, Leçons sur la Géométrie, Paris, 1883, vol. 3, p. 463. 
are furnished by enlarged Cremona point transformations, Cremona line transformations, polar reciprocation, and combinations of these. That birational contact transformations, other than these types, exist was demonstrated by Fano who gave some examples. * Groups of birational contact transformations were first investigated by Autonne, who determined the finite linear and finite quadratic groups of birational transformations in the plane. $\dagger$

It is the purpose of this paper to construct and study an infinite mixed group of birational contact transformations in $S_{2}$. It will then be shown that an analogous group exists in $S_{n}$.

2. The abelian group of birational contact transformations. We begin with the consideration of a certain directrix equation

$$
\sum\left(y_{i} / x_{i}\right)^{1 / k}=0,
$$

$k$ an integer, $(|k|>1)$, which, it will be shown, determines a birational contact transformation belonging to a group of such transformations. In $\$ 4$ these transformations will be generalized to $r$-space, and in turn the directrix equations of the generalized transformations will be derived.

By virtue of (3) there corresponds to every point $y_{i}$ in the $y v$-plane a triangular-symmetric curve in the $x u$-plane.f Likewise, to every point $x_{i}$ in the $x u$-plane corresponds a triangular-symmetric curve in the $y v$-plane. To a generic curve in either plane corresponds the envelope of a family of triangular-symmetric curves in the other plane.

Differentiating (3), first under the assumption that the $y_{i}$ are constants and second that the $x_{i}$ are constants, we obtain

and

$$
\sum_{i=1}^{3} y_{i}^{1 / k} x_{i}^{-(1+k) / k} d x_{i}=0
$$

$$
\sum x_{i}^{-1 / k} y_{i}^{(1-k) / k} d y_{i}=0 .
$$

From (3) and (4) it follows that

* G. Fano, Trasformazione di contatto birazionali del piano, Rendiconti delle Reale Accademia Nazionale dei Lincei, (6), vol. 8 (1928), p. 445.

$\dagger$ L. Autonne, (1) Groupes d'ordre fini des substitutions linéaires de contact, Journal de Mathématique, (4), vol. 3 (1887); (2) Groupes d'ordre fini contenus dans le groupe quadratique crémonien, ibid., vol. 4 (1888), pp. 177, 407; (3) Autonne's investigations regarding groups in $S_{3}$ and $S_{n}$ which appear in Annales de l'Université de Lyon, Science et Médecine, vol. 16, 1905.

$\ddagger$ Triangular-symmetric curves are those of the type $\left(x_{1} / a_{1}\right)^{m}+\left(x_{2} / a_{2}\right)^{m}+\left(x_{3} / a_{3}\right)^{m}$ $=0$. See, for instance, G. Loria, Spezielle algebraische und transzendente ebene Kurven, 1910, vol. 1, p. 341. 


$$
\begin{aligned}
& \rho y_{1}=x_{1}^{k+1}\left(x_{2} d x_{3}-x_{3} d x_{2}\right)^{k}, \\
& \rho y_{2}=x_{2}^{k+1}\left(x_{3} d x_{1}-x_{1} d x_{3}\right)^{k}, \\
& \rho y_{3}=x_{3}^{k+1}\left(x_{1} d x_{2}-x_{2} d x_{1}\right)^{k} ;
\end{aligned}
$$

and from (3) and (5) that

$$
\begin{aligned}
& \rho^{\prime} x_{1}=y_{1}^{-(k-1)}\left(y_{2} d y_{3}-y_{3} d y_{2}\right)^{-k}, \\
& \rho^{\prime} x_{2}=y_{2}^{-(k-1)}\left(y_{3} d y_{1}-y_{1} d y_{3}\right)^{-k} \\
& \rho^{\prime} x_{3}=y_{3}^{-(k-1)}\left(y_{1} d y_{2}-y_{2} d y_{1}\right)^{-k} .
\end{aligned}
$$

Since

$$
\begin{aligned}
& u_{1}: u_{2}: u_{3}=(x d x)_{1}:(x d x)_{2}:(x d x)_{3}, \\
& v_{1}: v_{2}: v_{3}=(y d y)_{1}:(y d y)_{2}:(y d y)_{3},
\end{aligned}
$$

we find, by substituting in (6) and (7) and then solving for $v_{i}$ and $u_{i}$, that the equations of the birational contact transformation $T_{k}$ and of its inverse $T_{k}^{-1}$ are

$$
\begin{aligned}
\rho y_{i} & =x_{i}^{k+1} u_{i}^{k},
\end{aligned} \quad i=1,2,3,
$$

and

$$
\begin{array}{rlrl}
\rho^{\prime} x_{1} & =\left(y_{2} y_{3}\right)^{k-1}\left(v_{2} v_{3}\right)^{k}, & & \\
T_{k}^{-1}: & \rho^{\prime} x_{3} & =\left(y_{1} y_{2}\right)^{k-1}\left(v_{1} v_{2}\right)^{k}, & \\
\sigma^{\prime} u_{i} & =v_{i}^{k+1} y_{i}{ }^{k}, & i=1,2,3 .
\end{array}
$$

From the equations of $T_{k}$ it can be easily verified that

$$
\begin{aligned}
\rho \sigma \sum v_{i} y_{i} & =\left(x_{1} x_{2} x_{3}\right)^{k}\left(u_{1} u_{2} u_{3}\right)^{k-1} \sum u_{i} x_{i}, \\
\rho \sigma \sum v_{i} d y_{i} & =\left(x_{1} x_{2} x_{3}\right)^{k}\left(u_{1} u_{2} u_{3}\right)^{k-1}\left[(k+1) \sum u_{i} d x_{i}+k \sum x_{i} d u_{i}\right], \\
\rho \sigma \sum y_{i} d v_{i} & =-\left(x_{1} x_{2} x_{3}\right)^{k}\left(u_{1} u_{2} u_{3}\right)^{k-1}\left[k \sum u_{i} d x_{i}+(k-1) \sum x_{i} d u_{i}\right] .
\end{aligned}
$$

Evidently $T_{k}$ is a birational contact transformation when $k=0,1$, so that the restriction previously put on $k$ may be removed.

The triangular-symmetric curve

$$
\left(a_{1} x_{1}\right)^{p}+\left(a_{2} x_{2}\right)^{p}+\left(a_{3} x_{3}\right)^{p}=0
$$

is transformed by $T_{k}$ into another triangular-symmetric curve, namely,

$$
\sum\left(a_{i} y_{i}\right)^{p /(k p+1)}=0
$$


hence $T_{k}$ leaves a three-parameter family of triangular-symmetric curves invariant. If $p^{\prime}=p /(k p+1)$, the indices* of $(8)$ and $\left(8^{\prime}\right)$ are connected by the equation

$$
\frac{1}{p^{\prime}}-\frac{1}{p}=k .
$$

Since $T_{0}$ transforms a generic element into itself, it is an identical transformation and will be represented by the symbol $I$. We note, furthermore, that

$$
T_{k}^{-1}=T_{-k}, \quad T_{k} T_{-k}=I, \quad T_{k} T_{m}=T_{m} T_{k}=T_{k+m} .
$$

Consequently, the transformations $T_{k},(k=\cdots,-2,-1,0,1$, $2, \cdots)$, form a commutative group, $G$.

THEOREM 1. The set of $\infty^{1}$ transformations $T_{k}$ ( $k$ an integer) forms $a$ discrete abelian group of birational contact transformations that leave a family of $\infty^{3}$ triangular-symmetric curves invariant.

Let the vertices of the fundamental triangle be $A_{1}(1,0,0)$, $A_{2}(0,1,0), A_{3}(0,0,1)$, and let $(x, u)$ be a generic line element. If $A, B, C$ represent lines joining $x$ to $A_{1}, A_{2}, A_{3}$, respectively, and if $D$ represents $u$, then the coordinates of these four lines are given by

$$
\begin{aligned}
& A: \quad 0, x_{3},-x_{2} \text {, } \\
& B: \quad x_{3}, 0,-x_{1}, \\
& C: \quad x_{2},-x_{1}, 0 \text {, } \\
& D: \quad u_{1}, u_{2}, u_{3} \text {. }
\end{aligned}
$$

Evidently

$$
x_{3} C=-x_{1} A+x_{2} B, \quad x_{3} D=u_{2} A+u_{1} B .
$$

Therefore

$$
R(A B, C D)=-\frac{x_{2} u_{2}}{x_{1} u_{1}} .
$$

If $(y, v)$ is the line element that corresponds to $(x, u)$ by virtue of $T_{k}$, and if $A^{\prime}, B^{\prime}, C^{\prime}$ represent lines $y A_{1}, y A_{2}, y A_{3}$, respectively, while $D^{\prime}$ represents $v$, then the coordinates of $A^{\prime}, B^{\prime}, C^{\prime}, D^{\prime}$ are as follows:

$$
\begin{aligned}
& A^{\prime}: \quad 0, x_{3}{ }^{k+1} u_{3}{ }^{k},-x_{2}{ }^{k+1} u_{2}{ }^{k}, \\
& B^{\prime}: \quad x_{3}{ }^{k+1} u_{3}^{k}, 0,-x_{1}^{k+1} u_{1}^{k}, \\
& C^{\prime}: \quad x_{2}^{k+1} u_{2}^{k},-x_{1}^{k+1} u_{1}^{k}, 0, \\
& D^{\prime}: \quad\left(x_{2} x_{3}\right)^{k}\left(u_{2} u_{3}\right)^{k-1},\left(x_{3} x_{1}\right)^{k}\left(u_{3} u_{1}\right)^{k-1},\left(x_{1} x_{2}\right)^{k}\left(u_{1} u_{2}\right)^{k-1} .
\end{aligned}
$$

* G. Loria calls $p$ the index of the curve (8); loc. cit., p. 329. 
Therefore

$$
\begin{aligned}
x_{3}^{k+1} u_{3}^{k} C^{\prime} & =-x_{1}^{k+1} u_{1}^{k} A^{\prime}+x_{2}^{k+1} u_{2}^{k} B^{\prime} \\
x_{3} u_{3} D^{\prime} & =x_{1}^{k} u_{1}^{k-1} A^{\prime}+x_{2}^{k} u_{2}^{k-1} B^{\prime} .
\end{aligned}
$$

Consequently,

$$
R\left(A^{\prime} B^{\prime}, C^{\prime} D^{\prime}\right)=-\frac{x_{2} u_{2}}{x_{1} u_{1}}=R(A B, C D) .
$$

In the same way it can be shown that the cross ratio that $x$ forms with the points at which $u$ intersects the sides of the fundamental triangle is equal to the cross ratio that the point $y$ of the corresponding line element forms with the points at which $v$ intersects the sides of the fundamental triangle.

THEOREM 2. Every transformation in the group $G$ sets up a one-toone correspondence between the line elements of the plane in such manner that, if $(x, u)$ and $(y, v)$ are corresponding elements, (1) the cross ratio that $u$ forms with the lines joining $x$ to the vertices of the fundamental triangle equals the cross ratio that $v$ forms with the lines joining $y$ to the vertices of the fundamental triangle, and dually, (2) the cross ratio that $x$ forms with the points at which $u$ intersects the sides of the fundamental triangle equals the cross ratio that $y$ forms with the points at which $v$ intersects the sides of the fundamental triangle.

The $W$ curves

$$
x_{1}^{a_{1}} x_{2}^{a_{2}} x_{3}^{a_{3}}=C, \quad a_{1}+a_{2}+a_{3}=0,
$$

may be regarded as limits of the triangular-symmetric curves

$$
a_{1} x_{1}{ }^{m}+a_{2} x_{2}{ }^{m}+a_{3} x_{3}{ }^{m}=0, \quad a_{1}+a_{2}+a_{3}=0, \quad a_{i} \text { real, }
$$

when $m \rightarrow 0$. $^{*}$ Moreover, each of these $W$ curves has the property that the cross ratio of any one of its points and the three points of intersection of the tangent at the point with the sides of the fundamental triangle is constant for the curve; and dually, the cross ratio of any one of its tangents and the three lines joining the point of tangency to the vertices of the fundamental triangle is constant for the curve. $\dagger$ 130.

* Cesàro-Kowalewski, Vorlesungen über natürliche Geometrie, Leipzig, 1901, p.

$\dagger$ The cross ratio associated with any $W$ curve is independent of $C$, its value being $-a_{i} / a_{l},(i \neq j)$, where the subscripts depend on the sequence of the elements forming the cross ratio. To a given cross ratio correspond $\infty^{1}$ curves of the family (9), so that (9) constitutes a two-parameter family. It should be noted that only the ratios of the $a_{i}$ and not the $a_{i}$ themselves are significant as parameters. See F. Klein, Vorlesungen über Höhere Geometrie, Berlin, 1926, p. 170. 
Consequently we can add the following corollary to Theorem 2:

COROLLARY. The birational contact transformations of group $G$ transform each member of a two-parameter family of $W$ curves into another member of the same family possessing the same characteristic cross ratio.

3. The $\infty^{1}$ involutory birational contact transformations. The directrix equation

$$
\sum_{i=1}^{3}\left(y_{i} / u_{i}\right)^{1 / k}=0
$$

determines a birational contact transformation $S_{k}$ the equations of which are obtained in the same manner as were the equations of $T_{k}$ :

$$
\begin{aligned}
& \rho y_{i}=x_{i}^{k} u_{i}^{k+1}, \\
& S_{k}: \quad \sigma v_{1}=\left(x_{2} x_{3}\right)^{k-1}\left(u_{2} u_{3}\right)^{k}, \quad \sigma v_{2}=\left(x_{3} x_{1}\right)^{k-1}\left(u_{3} u_{1}\right)^{k} \text {, } \\
& \sigma v_{3}=\left(x_{1} x_{2}\right)^{k-1}\left(u_{1} u_{2}\right)^{k}, \quad i=1,2,3 \text {, }
\end{aligned}
$$

and

$$
\begin{aligned}
\rho^{\prime} x_{i} & =y_{i}^{k} v_{\imath}^{k+1}, & & \\
S_{k}^{-1}: & \sigma^{\prime} u_{1} & =\left(y_{2} y_{3}\right)^{k-1}\left(v_{2} v_{3}\right)^{k}, \quad \sigma^{\prime} u_{2}=\left(y_{3} y_{1}\right)^{k-1}\left(v_{3} v_{1}\right)^{k}, & \\
\sigma^{\prime} u_{3} & =\left(y_{1} y_{2}\right)^{k-1}\left(v_{1} v_{2}\right)^{k}, & & i=1,2,3 .
\end{aligned}
$$

From the equations of $S_{k}$ it follows that

$$
\rho \sigma \sum y_{i} v_{i}=\left(x_{1} x_{2} x_{3}\right)^{k-1}\left(u_{1} u_{2} u_{3}\right)^{k} \sum x_{i} u_{i},
$$

and

$$
\begin{aligned}
& \rho \sigma \sum y_{i} d v_{i}=-\left(x_{1} x_{2} x_{3}\right)^{k-1}\left(u_{1} u_{2} u_{3}\right)^{k}\left[(k-1) \sum u_{i} d x_{i}+k \sum x_{i} d u_{i}\right] \\
& \rho \sigma \sum v_{i} d y_{i}=\left(x_{1} x_{2} x_{3}\right)^{k-1}\left(u_{1} u_{2} u_{3}\right)^{k}\left[k \sum u_{i} d x_{i}+(k+1) \sum x_{i} d u_{i}\right] .
\end{aligned}
$$

It is apparent that $S_{k}$ is an involutory birational contact transformation. By means of $S_{k}$ the $\infty^{3}$ triangular-symmetric curves (8) are transformed into

$$
\sum_{i=1}^{3}\left(y_{i} / a_{i}\right)^{p /(p k+p-1)}=0 .
$$

If the index of $\left(8^{\prime \prime}\right)$ is represented by $p^{\prime}$, it is seen that the indices of a triangular-symmetric curve and its transform by $S_{k}$ are connected by the equation

$$
\frac{1}{p}+\frac{1}{p^{\prime}}=k+1
$$


Four triangular-symmetric curves are left invariant by $S_{k}$, namely,

$$
\sum_{i=1}^{3} \pm\left(x_{i}\right)^{2 /(k+1)}=0 .
$$

Regard $S_{k}$ as a substitution, and let $S_{k} S_{n}$ represent the effect of substitution $S_{k}$ followed by $S_{n}$. Let $P$ represent the enlarged polarity $\rho y_{i}=u_{i}, \sigma v_{i}=x_{i}$. Then it easy to show that

$$
\begin{aligned}
S_{k} S_{n} & =T_{k-n}, & S_{k}^{2} & =I, \quad S_{0}=P, \\
S_{n} T_{k} & =S_{n-k}, & T_{k} S_{n} & =T_{n} S_{k}=S_{k+n}, \\
S_{k} T_{k} & =P, & S_{k} P & =T_{k}, \quad P S_{k}=T_{-k}, \\
P S_{k} P & =S_{-k}, & S_{n} T_{k} S_{n} & =T_{-k} .
\end{aligned}
$$

The $\infty^{1}$ birational contact transformations $S_{k}$, ( $k$ an integer), do not form a group, but the aggregate set of the $S_{k}$ and the $T_{n}$ form a mixed group $\Gamma$ of which $G$ is evidently an invariant subgroup.

THEOREM 3. The set of $\infty^{1}$ involutory birational contact transformations $S_{k}$ and the set of $\infty^{1}$ birational contact transformations $T_{n}$ together form a mixed group $\Gamma$ which contains $G$ as an invariant subgroup.

For any integer $c$ the set of transformations $T_{k c}$ obviously forms a group $G_{1}$, a subgroup of $G$. Moreover, the set of transformations in $G_{1}$ and the set $S_{k c}$ together form a group $\Gamma_{1}$, a subgroup of $\Gamma$. If we set up a one-to-one correspondence between the members of $\Gamma_{1}$ and $\Gamma$, such that $T_{k c}$ and $T_{k}$ correspond to one another and $S_{k c}$ and $S_{k}$ also correspond to one another for all integral values of $k$, it is seen, in view of the relationships given in (10), that $\Gamma_{1}$ and $\Gamma$ are isomorphic and so are $G_{1}$ and $G$.

If $(x, u)$ and $(y, v)$ are a pair of corresponding line elements with respect to $S_{k}$, it can be shown, as it was for $T_{k}$, that the cross ratio formed by $u$ and the lines joining $x$ to the vertices of the fundamental triangle is equal to the cross ratio formed by $v$ and the three lines joining $y$ to the vertices of the fundamental triangle, and a similar result holds in the dual case.

THEOREM 4. Every transformation in $\Gamma$ preserves the cross ratio formed by the line of a generic line element and the joins of its point with the vertices of the fundamental triangle; and dually, every transformation in $\Gamma$ preserves the cross ratio formed by the point of a generic line element and the intersections of its line with the sides of the fundamental triangle.

It was shown above that the $T_{k}$ leave the two-parameter family of 
$W$ curves (9) invariant. For the same reason the $S_{k}$ leave the same family invariant.* Consequently, we add the following corollary:

COROLlaRy. The transformations in $\Gamma$ leave a two-parameter family of $W$ curves invariant.

It is interesting to observe that $S_{n}$ and $T_{n}$ are determined by other directrix equations in addition to those indicated above. $\dagger$ For instance, the directrix equations

$$
\sum\left(y_{i} x_{i}\right)^{1 /(n+1)}=0, \quad \sum\left(v_{i} u_{i}\right)^{1 /(1-n)}=0, \quad \sum\left(v_{i} / x_{i}\right)^{-1 / n}=0
$$

determine $S_{n}$, whereas

$$
\sum\left(y_{i} u_{i}\right)^{1 /(n+1)}=0, \quad \sum\left(v_{i} x_{i}\right)^{1 /(1-n)}=0, \quad \sum\left(v_{i} / u_{i}\right)^{-1 / n}=0
$$

determine $T_{n}$.

4. Extension to higher space. The transformations $T_{n}$ and $S_{n}$ have analogs in higher spaces. Thus in $r$-space, the analog of $T_{n}$ is given by

$$
\bar{T}_{n}: \quad \rho y_{i}=x_{i}{ }^{n+1} u_{i}{ }^{n}, \quad \sigma v_{i}=\frac{1}{x_{i}{ }^{n} u_{i}-1}, \quad i=1,2, \cdots, r+1,
$$

and the analog of $S_{n}$ is given by

$$
\bar{S}_{n}: \quad \rho y_{\imath}=x_{i}{ }^{n} u_{i}{ }^{n+1}, \quad \sigma v_{i}=\frac{1}{x_{i}{ }^{n-1} u_{i}{ }^{n}}, \quad i=1,2, \cdots, r+1 .
$$

From the first $r+1$ equations in (11) it follows that $\rho y_{i} / x_{i}=x_{i}^{n} u_{i}^{n}$. Therefore, using the relationship $\sum u_{i} x_{i}=0$, we obtain a directrix equation of $\bar{T}_{n}$ in the form

$$
\sum_{i=1}^{r+1}\left(y_{i} / x_{i}\right)^{1 / n}=0 .
$$

From the second set of $r+1$ equations in (11) the directrix equation of $\bar{T}_{n}$ in line coordinates is found to be

$$
\sum_{i=1}^{r+1}\left(u_{i} / v_{i}\right)^{1 / n}=0 .
$$

Directrix equations of $\bar{S}_{n}$ in mixed point and line coordinates, found

* A detailed proof appears below in connection with a discussion of these transformations in $r$-space.

$\dagger$ Autonne, Journal de Mathématique, vol. 4 (1888), shows that every contact transformation in mixed coordinates is determined by four directrix equations (primordiales), namely, point-punctual, point-linear, line-punctual, line-linear. 
in the same manner, are

and

$$
\sum_{i=1}^{r+1}\left(y_{i} / u_{i}\right)^{1 / n}=0
$$

$$
\sum_{i=1}^{r+1}\left(x_{i} / v_{i}\right)^{1 / n}=0 .
$$

The directrix equations of each of these transformations can be expressed in two other forms, as was indicated above in the planar case.

The aggregate of birational contact transformations, $\bar{T}_{n}$ and $\bar{S}_{n}(n$ an integer), can be shown to constitute a group $\bar{\Gamma}$ that has an invariant abelian subgroup $\bar{G}$ composed of the transformations $\bar{T}_{n}$. The transformation $\bar{S}_{n}$ is involutory, interchanges the $\infty^{r+1}$ varieties in (13), replacing

$$
\sum_{i=1}^{r+1}\left(a_{i} x_{i}\right)^{1 / p}=0
$$

by

$$
\sum\left(y_{i} / a_{i}\right)^{p /(p n+p-1)}=0,
$$

and leaves each variety in the set

$$
\sum \pm\left(x_{i}\right)^{2 /(n+1)}=0
$$

invariant. On the other hand, $\bar{T}_{n}$ transforms the family (13) into itself, replacing (13) by

$$
\sum\left(a_{i} y_{i}\right)^{p /(n p+1)}=0 .
$$

Because these statements can be proved in the same way that their analogues regarding $S_{n}$ and $T_{n}$ in the plane were proved, no verification is offered.

Let us consider the $\infty^{r}$ varieties

$$
F \equiv \prod_{i=1}^{r+1} y_{i}^{a_{i}}=K, \quad \sum_{1}^{r+1} a_{i}=0
$$

$K$ constant. This family is evidently a generalization in $r$-space of the $W$ curves discussed above. ${ }^{*}$ It will be proved that this family is left invariant by the transformations in $\bar{\Gamma}$. First we show that $\bar{T}$ leaves (14) invariant.

* Surfaces of this type in three-space were investigated by Lie and Klein, Comptes Rendus de l'Académie des Sciences, 1870. 
Let $v_{i},(i=1,2, \cdots, r+1)$, be the coordinates of the hyperplane tangent to $F$ at $y_{i} ;$ then

$$
\alpha v_{i}=\frac{\partial F}{\partial y_{i}}=\frac{a_{i} K}{y_{i}},
$$

where $\alpha$ is a constant of proportionality. Eliminating the $v_{i}$ from (15) and from

$$
\sigma v_{i}=\frac{1}{x_{i}^{n} u_{i}^{n-1}}
$$

we obtain

$$
\alpha^{\prime} y_{i}=a_{i} x_{i}{ }^{n} u_{i}^{n-1} K \text {. }
$$

If we let the new constant of proportionality absorb the constant $K$, and if we drop the prime, this equation becomes

$$
\alpha y_{i}=a_{i} x_{i}^{n} u_{i}^{n-1} .
$$

The elimination of the $u_{i}$ from (16) and $\rho y_{i}=x_{i}{ }^{n+1} u_{i}{ }^{n}$ results in

$$
\rho^{\prime} y_{i}=a_{i}{ }^{n} x_{i} .
$$

Substituting from (17) in (14) we obtain the transform of (14), namely

$$
\prod_{1}^{r+1} a_{i}^{n a_{i}} \prod_{1}^{r+1} x_{i}^{a_{i}}=K, \quad \sum_{1}^{r\lrcorner 1} a_{i}=0
$$

In the same way it can be proved that $\bar{S}_{n}$ transforms (14) into

$$
K \prod_{1}^{r+1} x_{i}^{a_{i}}=\prod_{1}^{r+1} a_{i}^{a_{i}(n+1)}, \quad \sum_{1}^{r+1} a_{i}=0,
$$

so that all the members of $\bar{\Gamma}$ transform this family into itself.*

New York City

* Continuous (Lie) groups in which the discrete groups $\Gamma$ and $\bar{\Gamma}$ are immersed will be considered in a subsequent paper. 